\title{
Aplicación de la CITES para especies usadas como carne de monte y sus impactos sobre los medios de vida en Colombia
}

\author{
Gomez Juanita, Restrepo Sebastián y van Vliet Nathalie
}

\section{Puntos clave}

- El Centro Internacional para la Investigación Forestal (CIFOR) y la Secretaría de la Convención sobre el Comercio Internacional de Especies Amenazadas de Fauna y Flora Silvestres (CITES), desarrollaron en 2016 una guía que permite a los países signatarios del convenio, efectuar una evaluación rápida del comercio internacional de carne de monte en sus territorios, al igual que un diagnóstico general del impacto de la inclusión de especies en los Apéndices de la CITES y la identificación de medidas de mitigación.

- Entre enero y junio de 2016, se llevó a cabo un estudio de caso en Colombia para aplicar la guía. La metodología aplicada consistió en una revisión bibliográfica; consultas a instituciones y autoridades ambientales; encuestas a expertos en el tema; visitas de campo a lugares estratégicos; y talleres regionales con la participación de actores en la cadena de mercado, autoridades y expertos.

- En Colombia el comercio de carne de monte opera de forma transfronteriza, en lugares donde las condiciones geográficas dificultan un control institucional, además de localizarse en puntos geográficos específicos; se identificaron cuatro rutas internacionales, tres de ellas en la Amazonía y una en la región Caribe.

- La mayoría de las especies involucradas en el comercio internacional de carne de monte están incluidas en las listas de los Apéndices de la CITES: paca, guara, zaíno y tortugas. Por ello, la aplicación de la Convención podría generar impactos negativos en los medios de vida de los actores que hacen parte de las dinámicas de comercio transfronterizo.

- Los participantes en los talleres manifestaron que pese a la existencia de medidas alternativas, el comercio de carne de monte seguirá ocurriendo dado los bajos costos que representa, en comparación con cualquier forma de producción doméstica. Así mismo, el consumo de carne de monte en algunos contextos está fuertemente arraigado a la cultura local, lo cual dificulta el éxito de las medidas alternativas.

- La principal recomendación general que surge del presente estudio de caso es la de diferenciar el tratamiento del comercio transfronterizo a escala local por parte de comunidades de dos países vecinos (como es el caso de Colombia), del comercio de lujo a gran escala (ej. desde África Central hacia Europa, o desde África occidental hacia Estados Unidos), a efectos de medir los impactos en las comunidades por la aplicación de las disposiciones la CITES

\section{Introducción}

La Secretaría General de la Organización de Estados Americanos (OEA) y la Secretaría de la Convención sobre el Comercio Internacional de Especies Amenazadas de Fauna y Flora Silvestre (CITES), desarrollaron en 2015 una guía para la evaluación rápida de los efectos de la aplicación de las disposiciones de la CITES en los medios de subsistencia de las comunidades rurales pobres. Por su parte, el Grupo de Trabajo sobre Carne de Monte de la CITES ha reconocido que el comercio internacional de carne de monte en un contexto de ilegalidad puede representar una amenaza para las poblaciones silvestres de especies listadas en la CITES, al igual que para la seguridad alimentaria y los medios de vida de las comunidades que dependen de la fauna silvestre.

Teniendo en cuenta los problemas asociados al comercio internacional de carne de monte en un marco de ilegalidad, la Secretaría de la CITES y CIFOR desarrollaron en 2016, una guía sobre carne de monte y medios de vida, basada en la guía de la CITES y la OEA. Este trabajo tiene como objetivo permitir a los países parte del convenio efectuar una evaluación rápida del comercio internacional de carne de monte en sus territorios, considerando las zonas geográficas donde opera el comercio, las especies comercializadas y los actores implicados en la cadena de mercado. Así mismo, la guía permite realizar un diagnóstico general del impacto que genera la inclusión de especies en los Apéndices de la CITES y la identificación de medidas de mitigación.

Con el propósito de aplicar la metodología propuesta por la guía CITES sobre carne de monte y medios de vida, CIFOR llevó a cabo un estudio de caso en Colombia entre los meses de enero y junio de 2016, en coordinación con las autoridades de CITES en Colombia. Durante este periodo se realizó un diagnóstico rápido de las rutas, actores y especies comercializadas como carne de monte desde o hacia Colombia. Así mismo, se analizaron los impactos de las regulaciones de la CITES sobre los medios de vida de las personas que basan su sustento en el comercio de carne de monte, al igual que las medidas para mitigar estos efectos. 
El estudio, cuya metodología se basó en la guía del CITES, se llevó a cabo en diferentes etapas. En primer lugar se realizó una revisión bibliográfica a la par de una consulta a las autoridades ambientales nacionales, regionales y locales, y encuestas a expertos en el tema. Una vez identificados los posibles puntos de comercio internacional, se realizaron 12 visitas de campo, focalizando la atención en las fronteras del país y en los principales puertos marítimos. Al concluir las visitas se seleccionaron los lugares con un flujo significativo de comercio internacional de carne de monte y se llevaron a cabo talleres con expertos, autoridades locales y actores de la cadena de comercio para formular alternativas al comercio de carne de monte. Los talleres fueron desarrollados en Leticia y Maicao.

\section{Principales resultados}

\section{a) Rutas de comercio internacional 0 transfronterizo:}

El comercio internacional de carne de monte en Colombia no opera a través de largas cadenas de comercialización.

Por el contrario, se limita a un comercio con fines de subsistencia en cadenas cortas, entre países vecinos. Muchas veces se trata de un comercio entre un lado y otro de un río, que marca una frontera oficial pero no una frontera social y cultural. Estas rutas son geográfica y temporalmente dinámicas, puesto que dependen de ciertos factores coyunturales (ej. cierre oficial de la frontera con Venezuela, o devaluación o valorización de la moneda con respecto al país fronterizo). El estudio de caso evidenció la existencia de cuatro rutas principales de comercio transfronterizo en Colombia, las tres primeras ubicadas en la Amazonía y la última en la región Caribe.

- Desde la parte brasilera y peruana del río Javarí, hacia Leticia (Colombia).

- Desde Puerto Ospina (Colombia), hacia Puerto del Carmen (Ecuador).

- Desde Tarapacá (Colombia) hacia Brasil, para el comercio de tortuga charapa (Podocnemis expansa), en la temporada de aguas bajas.

- Desde Venezuela hacia Maicao (Colombia), para el comercio de tortugas marinas (Chelonioidea sp.)

\section{b) Actores en la cadena de mercado:}

Las cadenas de mercado transfronterizas, se componen de cazadores, intermediarios, vendedores y consumidores. La mayoría de estos actores caza, vende o consume carne de monte de diferentes especies, de las cuales algunas están listadas en los apéndices de la CITES. Gran parte del comercio es nacional, pero también se da a través de las fronteras. Este estudio no estableció cantidades de carne de monte comercializada. Sin embargo, el número de actores involucrados y la estructura de las cadenas de mercado indican que el comercio es esporádico y oportunista para la mayoría de especies, con excepción de las tortugas marinas.

- Cazadores: La cacería de especies listadas en los apéndices de la CITES y destinadas al comercio transfronterizo es realizada principalmente por parte de hombres rurales de origen indígena o por colonos, pero también por parte de cazadores urbanos o periurbanos, que residen cerca a los principales puntos de entrada y salida de la carne de monte. Para la gran mayoría de cazadores, la cacería complementa otras actividades de subsistencia y es realizada principalmente con fines de consumo. Solo los excedentes son vendidos a familiares o clientes conocidos (intermediarios dentro de la cadena de mercado o directamente al consumidor final).

- Intermediarios: En algunos municipios como Leticia y Maicao, los intermediarios ejercen un rol fundamental en la cadena de mercado transfronteriza, generando ingresos importantes mediante la intermediación. Los intermediarios compran la carne de monte a los cazadores de un lado de la frontera y la comercializan a vendedores en la plaza de mercado, puestos ambulantes, y restaurantes, o directamente a consumidores conocidos del pais vecino.

- Vendedores: La carne de monte en las diferentes regiones de Colombia es vendida principalmente en puestos ambulantes, plazas de mercado, puertos y restaurantes. En algunos casos los vendedores se especializan en el comercio de carne de monte (ej. puestos de venta ambulantes de carne de tortuga en Maicao). Sin embargo, la mayoría de los vendedores diversifican su oferta con pescado y otro tipo de carnes producidas localmente (ej. los vendedores de la plaza de mercado de Leticia que complementan la venta de pescado de lujo, como el Pirarucú, con la venta de carne de monte).

- Consumidores: Los consumidores finales adquieren la carne de monte en las plazas de mercado para prepararla en casa o la consumen en los restaurantes y puestos ambulantes de comida. La cantidad de consumidores de carne de monte en cada zona del país, varía dependiendo de la preservación de tradiciones culturales, de la disponibilidad de otro tipo de proteínas y de las posibilidades de acceso a estas, dependiendo de su valor económico.

\section{c) Principales especies comercializadas en las rutas transfronterizas o internacionales}

\section{La tabla 1 señala las especies presentes en las cadenas de comercio fronterizo.}

\begin{tabular}{|c|c|c|c|}
\hline $\begin{array}{l}\text { Nombre } \\
\text { común }\end{array}$ & $\begin{array}{l}\text { Nombre } \\
\text { científico }\end{array}$ & $\begin{array}{l}\text { Regiones en las } \\
\text { que se comercializa } \\
\text { internacionalmente }\end{array}$ & $\begin{array}{l}\text { Apéndice } \\
\text { CITES }\end{array}$ \\
\hline Chuigüiro & $\begin{array}{l}\text { Hydrochoerus } \\
\text { hydrochaeris }\end{array}$ & $\begin{array}{l}\text { Orinoquía, Caribe, } \\
\text { Amazonas }\end{array}$ & / \\
\hline $\begin{array}{l}\text { Lapa, paca, } \\
\text { boruga, } \\
\text { guagua }\end{array}$ & $\begin{array}{l}\text { Cuniculus } \\
\text { paca }\end{array}$ & $\begin{array}{l}\text { Orinoquía, Caribe, } \\
\text { Amazonas }\end{array}$ & III \\
\hline \multirow[t]{2}{*}{ Cajuche, zaíno } & Pecari tajacu & \multirow{2}{*}{$\begin{array}{l}\text { Orinoquía, Caribe, } \\
\text { Amazonas }\end{array}$} & II \\
\hline & Tayassu pecari & & II \\
\hline Picure, guara & $\begin{array}{l}\text { Dasyprocta } \\
\text { punctata }\end{array}$ & Orinoquía, Caribe & III \\
\hline $\begin{array}{l}\text { Tortuga } \\
\text { morrocoy }\end{array}$ & $\begin{array}{l}\text { Chelonoidis } \\
\text { carbonaria }\end{array}$ & $\begin{array}{l}\text { Orinoquía, Caribe, } \\
\text { Amazonas }\end{array}$ & II \\
\hline $\begin{array}{l}\text { Tortuga } \\
\text { morrocoy }\end{array}$ & $\begin{array}{l}\text { Chenoloidis } \\
\text { denticulata }\end{array}$ & Amazonas & II \\
\hline Tortuga carey & $\begin{array}{l}\text { Eretmochelys } \\
\text { imbricata }\end{array}$ & Caribe & I \\
\hline Tortuga laúd & $\begin{array}{l}\text { Dermochelys } \\
\text { coriacea }\end{array}$ & Caribe & I \\
\hline Tortuga boba & Caretta caretta & Caribe & I \\
\hline Tortuga verde & $\begin{array}{l}\text { Chelonia } \\
\text { mydas }\end{array}$ & Caribe & I \\
\hline
\end{tabular}




\section{d) Afectaciones a los medios de vida rurales por la aplicación de la CITES}

La importancia del comercio transfronterizo de carne de monte (de especies listadas en los apéndices de la (ITES) para los medios de vida, varía entre las diferentes comunidades rurales del país. En la mayoría de los casos, el comercio transfronterizo no puede disociarse de las dinámicas del comercio nacional de carne de monte, puesto que involucra a los mismos actores. En Colombia, la venta de carne de monte es ilegal a nivel nacional y la venta internacional es regulada por la CITES. Sin embargo, los actores continúan operando en la ilegalidad y complementan de manera significativa sus ingresos con el comercio clandestino de carne de monte. En general, la venta de carne de monte no representa la actividad económica principal de lo actores, sin embargo genera ingresos importantes en una economía diversificada. Dada la importancia del uso de carne de monte no solo como fuente de ingreso o de alimento, sino también como parte integrante de las culturas locales, la dependencia de este recurso no puede medirse únicamente en términos económicos.

\section{e) Medidas de mitigación}

De los talleres llevados a cabo en Leticia y Maicao, los participantes identificaron las siguientes medidas alternativas para mitigar los efectos de la aplicación de la CITES en los medios de vida de las comunidades que dependen del comercio de carne de monte.

\section{Alternativas para los cazadores:}

- Reemplazar la cacería por pesca, donde ya hay un mercado establecido.

- Promover la producción y comercio de productos agrícolas tradicionales, tanto para la alimentación como para el comercio.

- Promover la producción y venta de artesanías.

- Desarrollar actividades de ecoturismo en torno a los modos de vida indígenas y locales.

- Realizar proyectos de fortalecimiento de actividades productivas basados en la tradición.

- Promover el uso y manejo sostenible de los recursos naturales, a través de programas que incluyan la participación de los actores sociales en el monitoreo y control.

\section{Alternativas para los comerciantes:}

- Establecer la posibilidad legal de comercializar a nivel nacional una lista de especies no protegidas, bajo un plan de manejo sostenible.

- $\quad$ Establecer temporadas para la venta legal de ciertas especies.

- Desarrollar programas de cría y reproducción en cautiverio.

\section{Conclusiones}

El comercio internacional de carne de monte en Colombia, es transfronterizo y está concentrado en puntos geográficos específicos, la complejidad geográfica del territorio colombiano conlleva a que el comercio se dé en su mayoría a través de fronteras naturales (ej. ríos), entre comunidades vecinas, donde el control estatal es escaso o nulo. En estos contextos el comercio internacional es realmente un comercio local, que ocurre entre las fronteras de dos países. Los intercambios transfronterizos operan en puntos geográficos específicos, principalmente en tres lugares de la región amazónica, y en uno de la región Caribe. Esta forma de comercio internacional difiere sustancialmente del comercio a larga distancia, que se da por ejemplo desde África hacia Francia y Estados Unidos (Bair Brake et al. 2014; Chaber et al. 2010).

La falta de una definición oficial de "carne de monte" por parte de la CITES, podría dejar por fuera algunas especies que se comercializan desde Colombia con fines de consumo; algunas definiciones de carne de monte (ej. Nasi et al, 2008) limitan el término a la fauna terrestre, con lo cual se dejaría por fuera especies como las tortugas marinas. Sin embargo, el comercio de estas especies sigue patrones similares a aquellos observados para las especies terrestres consideradas como carne de monte.

\section{Debilidad institucional en el control del comercio internacional de carne de monte, y dificultad en la} aplicación de la ley en algunas zonas del país la presencia institucional es escasa, lo cual dificulta el control policial y judicial del comercio de carne de monte. Por otro lado, a menudo resulta un reto para las autoridades locales establecer distinciones precisas entre el uso de subsistencia, uso comercial nacional y uso comercial internacional. Al respecto, cabe tener en cuenta que los límites entre estos conceptos resultan difusos en el contexto de las realidades de los asentamientos rurales ubicados en zonas de frontera.

Debilidad de las alternativas de comercio de carne de monte, los participantes en los talleres manifestaron que pese a que existan medidas alternativas, el comercio de carne de monte seguirá ocurriendo dado los bajos costos que representa, en comparación con cualquier forma de producción doméstica. Así mismo, el consumo de carne de monte en algunos contextos está fuertemente arraigado a la cultura local, lo cual dificulta el éxito de las medidas alternativas.

\section{Recomendaciones}

- Diferenciar el comercio transfronterizo a escala local por parte de comunidades rurales del comercio de productos de lujo a gran escala en las regulaciones de la CITES, con el propósito de medir y mitigar los impactos potenciales en los medios de vida locales.

- Examinar la posibilidad de establecer una definición oficial del término carne de monte en el marco de la CITES, de modo tal que se facilite determinar el alcance de dicha definición. 
- $\quad$ Establecer mecanismos de cooperación interinstitucional entre los países fronterizos para el control del comercio de carne de monte, teniendo en cuenta las realidades sociales y culturales en las que opera este comercio. Para ello se recomienda a los países parte implementar la guía CITES sobre carne de monte y medios de vida, con el fin de proveer información que permita establecer acciones para el control, acordes al contexto.

- Desarrollar un sistema de control que permita monitorear tanto el uso y comercio de carne de monte, como las medidas de mitigación implementadas, para asegurar que las regulaciones de la CITES no produzcan impactos significativos en las comunidades rurales.

- Desarrollar programas que incluyan la participación de las comunidades locales en los sistemas de monitoreo del uso y comercio de carne de monte (monitoreo de fauna, cacería y comercio de carne de monte, entre otras opciones), mediante la implementación de incentivos económicos. Estos proyectos cobran especial relevancia para Colombia en el marco del proceso de postconflicto, para el cual se han planteado estrategias de aprovechamiento de la biodiversidad en la construcción de la paz (ONU, 2014).

\section{Agradecimientos}

Este trabajo ha sido posible gracias al aporte financiero de la CITES, UKAID, DFID y USAID a través de la Iniciativa de Invetigación sobre Carne de Monte de CIFOR.

Agradecemos la participación activa y el apoyo del Ministerio del Medio Ambiente y Desarrollo de colombia, los puntos focales CITES, las instituciones gubernamentales y académicas de Colombia y de los diferentes actores involucrados en el uso y comercio de carne de monte en las zonas fronterizas del país.
Este documento ha sido preparado por CIFOR en virtud del contrato con la Secretaría de la CITES y ha sido una realidad gracias al financiamiento de la Unión Europea. Las opiniones expresadas en este documento son de los autores. No reflejan necesariamente las opiniones de la Secretaría de la CITES, la Unión Europea, CIFOR, los editores, las instituciones para las que los autores trabajan, los financiadores o quienes revisaron el documento.

Las denominaciones geográficas incluídas en esta publicación no implican la expresión de ninguna opinión por parte de la Secretaría de la CITES o de la Unión Europea, sobre el estatus legal de ningún país, territorio o área, o respecto de las delimitaciones de sus fronteras. La responsabilidad de los contenidos del documento es exclusiva de sus autores.

\section{Referencias}

Bair-Brake H, Bell T, Higgins A, Bailey N, Duda M, Shapiro S, Eves HE, Marano N. y Galland G. (2014). Is that a rodent in your luggage? A mixed method approach to describe bushmeat importation into the United States. Zoonoses and public health, 61(2), 97-104.

Chaber A L., Allebone-Webb S, Lignereux Y, Cunningham A A, y Marcus Rowcliffe J. (2010). The scale of illegal meat importation from Africa to Europe via Paris. Conservation Letters, 3(5), 317-321.

Nasi R, Brown D, Wilkie D, Bennett E, Tutin C, van Tol G, y Christophersen T. (2008). Conservation and use of wildlife-based resources: the bushmeat crisis. Secretariat of the Convention on Biological Diversity, Montreal, and Center for International Forestry Research (CIFOR), Bogor. Series técnicas no. 33, 50 pág. Disponible en: https://www.cbd.int/doc/publications/cbd-ts-33-en.pdf

Organización de las Naciones Unidas, Colombia. 2014. Construcción de una Paz territorial estable, duradera y sostenible en Colombia. Disponible en: http:// www.co.undp.org/content/dam/colombia/docs/ MedioAmbiente/undp-co-pazyambiente-2015.pdf

\begin{tabular}{|c|c|c|}
\hline CGIAR & $\begin{array}{l}\text { PROGRAMA DE } \\
\text { INVESTIGACIÓN SOBRE } \\
\text { Bosques, Árboles y } \\
\text { Agroforestería }\end{array}$ & $\begin{array}{l}\text { Esta investigación fue realizada por CIFOR como parte del Programa de Investigación de CGIAR sobre } \\
\text { Bosques, Árboles y Agroforestería (CRP-FTA). El objetivo del programa es mejorar el manejo y uso de los } \\
\text { bosques, la agroforestería y los recursos genéticos de los árboles a lo largo del paisaje, desde bosques } \\
\text { hasta plantaciones. CIFOR dirige el programa CRP-FTA en asociación con Bioversity International, CATIE, } \\
\text { CIRAD, el Centro Internacional de Agricultura Tropical y el Centro Mundial de Agroforestería. }\end{array}$ \\
\hline
\end{tabular}

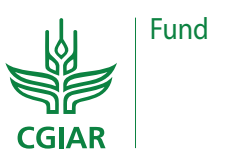

cifor.org

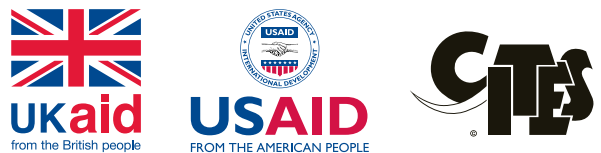

